\section{Risk informed design modification of dynamic thermal rating system}

ISSN 1751-8687

Received on 20th March 2015 Revised on 10th August 2015 Accepted on 14th August 2015 doi: 10.1049/iet-gtd.2015.0351 www.ietdl.org

\author{
Jiashen Teh ${ }^{凶}$, lan Cotton \\ Department of Electrical and Electronic Engineering, The University of Manchester, Oxford Road, Manchester, UK \\ E-mail: jiashen.teh-2@manchester.ac.uk
}

\begin{abstract}
Future grids will be operated much closer to their security limits due to the ever increasing power demands and the restrictions on land and space to build more transmission corridors. As one of the many smart grid technologies, the dynamic thermal rating (DTR) system of transmission lines offers the solution to increase line current capacity without infringing the conductor's maximum operating temperature. Hence, it has emerged as a popular solution for the aforementioned challenges. Owing to that, there is a strong need to assess the integrity of this technology. For this reason, this study presents a systematic framework for assessing the reliability and the risk of a DTR system. The research was carried out in two parts and is able to optimise the risk and the reliability (cost) of the DTR system. In addition, a weather estimation model is proposed to estimate the weather data when the DTR sensors are not functional. This is contrary to the existing literature which suggests that conservative assumptions be made. The results show that the proposed weather estimation model avoids overestimation of the DTR system risk.
\end{abstract}

\section{Nomenclature}

$Q_{\mathrm{c} i}$

$Q_{\mathrm{ri}}$

$Q_{\mathrm{r} i}$
$Q_{\mathrm{s} i}$
$T_{\mathrm{c} i}$

$T_{\mathrm{c} i}$
$T_{\mathrm{a} i}$

$T_{\mathrm{a} i}$

$\phi_{i}$

$\theta_{i}$
$I$

$R\left(T_{\mathrm{c}}\right)$
$P_{\mathrm{DTR}}^{\mathrm{Up}}$
$\mathrm{RS}$

RS

RS Al

STR Al

STR ST

STR $T$

$t$

$n_{\mathrm{Al}, \mathrm{ST}}$

$d_{\mathrm{Al}, \mathrm{ST}}$

$S_{\mathrm{Al}, \mathrm{ST}}$

AP

AP

Risk $_{\text {DTR }}$

Risk $_{\text {NoDTR }}$

Impact $_{\text {load }}$

Impact $_{\text {Anl }}$

Cost loadlos

Cost $_{\text {Anl }}$

$\mathrm{LC}_{i}$

LB

$\mathrm{PG}_{j}{ }^{\text {min }}$

$\mathrm{PG}_{j}^{\min }$

$\mathrm{PG}_{j}^{\max }$
$\mathrm{GB}$

GB

9

$\vartheta$
$X_{i j}$ convection heat loss at weather region $i(\mathrm{~W} / \mathrm{m})$ radiated heat loss at weather region $i(\mathrm{~W} / \mathrm{m})$ solar heat gain at weather region $i(\mathrm{~W} / \mathrm{m})$ conductor temperature at weather region $i\left({ }^{\circ} \mathrm{C}\right)$ ambient temperature at weather region $i\left({ }^{\circ} \mathrm{C}\right)$ wind speed at weather region $i(\mathrm{~m} / \mathrm{s})$ wind angle at weather region $i$ (degree) solar angle at weather region $i$ (degree) transmission line ampacity $(A)$ conductor AC resistance at $T_{\mathrm{c} i}(\Omega / \mathrm{m})$ up-state probability of DTR system remaining strength of conductor (\%) remaining strength of aluminium strands $(\%$ initial strength of aluminium strands (lbs.) initial strength of steel core (lbs.) initial strength of conductor (lbs.) elapsed time (hour)

number of aluminium/steel strand aluminium/steel strand diameter (in) rated strength of aluminium/steel strand (lbs.) loss of load probability due to DTR system failure annealing probability

total power system expected energy not served total risk of network with DTR system total risk of network without DTR system impact of loadloss

impact of line annealing

cost of loadloss

cost of line annealing

load curtailment at bus $i$ (MW)

load bus

generated power at bus $j(\mathrm{MW})$

minimum generated power at bus $j(\mathrm{MW})$

maximum generated power at bus $j(\mathrm{MW})$

generator bus

power demand at bus $i$ (MW)

bus voltage angle

reactance of line connecting bus $i$ to bus $j$
$\forall i \in \mathrm{LB}$
$\forall j \in \mathrm{GB}$
$\forall l \in B$
set of all load buses
set of all generator buses
set of all buses
set of all connected buses to bus $l$

\section{Introduction}

The static thermal rating (STR) of an overhead line is a rating that has been determined using the worst set of ambient weather conditions that can be expected during a particular season [1-3]. This effectively yield a seasonal thermal rating (SeTR), typically higher in winter months [1]. There are two disadvantages to these rating methods. First, they underestimate the line rating for the majority of time when wind speeds are higher or temperatures are lower of time when wind speeds are higher or temperatures are lower
than the assumed conditions. Second, while a low probability, the actual weather conditions might turn out worst than the conservative assumptions. Should this be the case there is a risk of overloading the lines.

The dynamic thermal rating (DTR) uses real-time meteorological data to compute transmission line ratings [3-6]. As it uses live weather conditions, it eliminates some of the problems that result from the use of the STR and SeTR techniques [7]. The usage of DTR is also supported by works that have clearly demonstrated the actual weather conditions are most of the time more desirable than the conservative assumptions [8-10]. It has been shown that a DTR system is able to usually increase transmission line capacity by $10-30 \%$ with improvement of $50 \%$ being possible in windy areas $[7,11]$. The installation cost of a DTR system is cheap when compared with major physical line upgrades and uses sensors that are commonplace [12-14]. A DTR system therefore offers an ability to delay new line investments, increase electrical transmission reliability and offer increased options in faulted network conditions.

The formation of IEEE Standard 738, for the calculation of overhead line steady-state and dynamic heat balance based on the meteorological conditions, is a testament to the importance of DTR technology [15]. Hence, several studies related to DTR have been undertaken. One study showed that the application of the DTR system in a power network enables evaluation of the conductor ageing process for better transmission line management

IET Gener. Transm. Distrib., pp. 1-8

This paper is a preprint of a paper accepted by [IET Generation Transmission \& Distribution] and is subject to Institution of Engineering and Technology Copyright. When the final version is published, the copy of record will be available at IET Digital Library 
[16]. Furthermore, an algorithm for strategic placement of DTR sensors has been proposed $[17,18]$. The uncertainty of DTR sensor measurement was also quantified by modifying IEEE Standard 738 with the fuzzy concept [19]. Finally, the impact of the DTR system on the reliability of the power network has been investigated [20].

All of the cited works are commended. However, none have ever assessed the integrity of DTR system. The advantages of the DTR system only exist if it is operating reliably - an overestimation of line rating could have a detrimental impact on transmission line as the maximum conductor temperature could be exceeded. As such, there is a need to assess the integrity of the DTR system and this paper presents a systematic framework to do that. The integrity consists of the reliability of DTR system and its risk when it is applied in a power network. Besides that, the line rating of all the above literature reverted to STR during the failure of DTR system. We have found that this overestimates the risk of DTR system and undermines the line capacity. Hence, the proposed framework also uses a weather estimation model to estimate the missing weather values of non-functional DTR sensors.

\section{Overview of DTR system integrity assessment framework}

An overview of the proposed methodology is given in Fig. 1. The process is divided into two parts. The first part of the methodology conducts a reliability assessment of DTR system and attempts to explore as many design options as possible. The design option are referring to various numbers of redundancies, failure and repair rates of DTR sensors. Starting with an initial DTR system design, it will undergo reliability sensitivity analyses to identify its critical components before any design modifications are made. Finally, all the identified design options are passed to the second part of the framework.

In the second part, the risk assessments of DTR system are conducted. The risk of DTR system is evaluated by examining its impact on the IEEE 24-bus reliability test network (RTN). Owing to the many combinations of DTR system and power network component statuses, the state space is explored using the Monte Carlo (MC) method. If any particular DTR sensors are not functional, the missing weather data is estimated using the multiple-linear regression (MLR) weather estimation model. Subsequently, the line rating values are evaluated based on the
IEEE Standard 738. On the contrary, the DTR sensors are normal and the line ratings are determined without the need of weather estimation. To determine the impact of DTR system on the RTN, DC-OPF was performed. During which, the expected energy not served (EENS) and line annealing properties are recorded and they represent the average risk of DTR system. Part 2 is repeated until all the DTR system designs have been assessed. Finally, the most suitable design is suggested (or selected) based on the optimisation among the risk values and the reliabilities of all the designs.

In Sections 4 and 5, all the models that made up the framework are described.

\section{Assessing DTR system reliability}

\subsection{Reliability model}

A transmission line that is spread across $n$ weather regions can be assumed to require $n$ monitoring station. In any weather region, it is assumed that the weather conditions remain constant. Air temperature is fairly constant over large areas and can generally be given by local meteorological data centres. The solar radiation angle and level have relatively small impact in the calculation of line ratings and the assumptions given by the IEEE Standard 738 can therefore be used [21]. Hence, in our DTR system model, each station has only wind speed and wind angle sensors. Note that in the initial design each DTR sensor has a redundancy. These sensors can either be functional or non-functional. In the functional state, the sensor is operating normally and delivers an accurate measurement. In the non-functional state, the sensor is either damaged or is mal-operating. During mal-operation, the measurements are inaccurate.

The reliability of the whole DTR system is determined using the reliability of the sensors. This is done through the use of the event tree analysis (ETA) as shown in Fig. 2 due to its fast computation and versatility [22]. Initially, the statuses of all the respective sensors are consolidated using the majority vote system. It works by validating the sensor values through comparisons with its redundant measurement values. The voting system is used as it has proven to be effective in various fields of nuclear and power engineering where sensing activities are required [23-27]. This process produces the statuses (up or down) of the wind speed and wind angle sensing abilities. Both of the sensing abilities need to be in the up-state in order to yield a normal operating monitoring

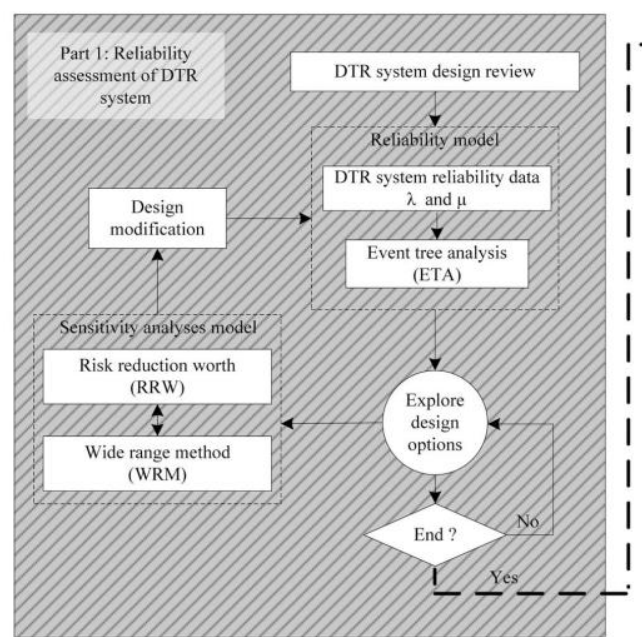

Fig. 1 Overview of DTR system reliability and risk assessment framework

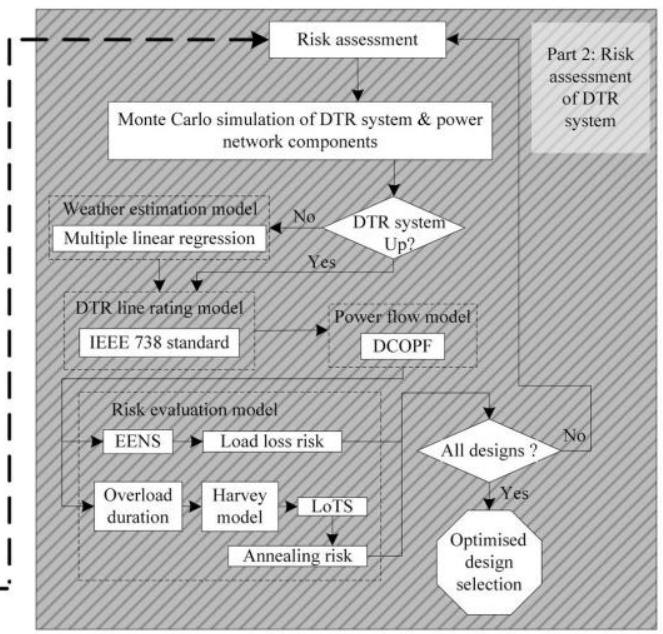

IET Gener. Transm. Distrib., pp. 1-8 


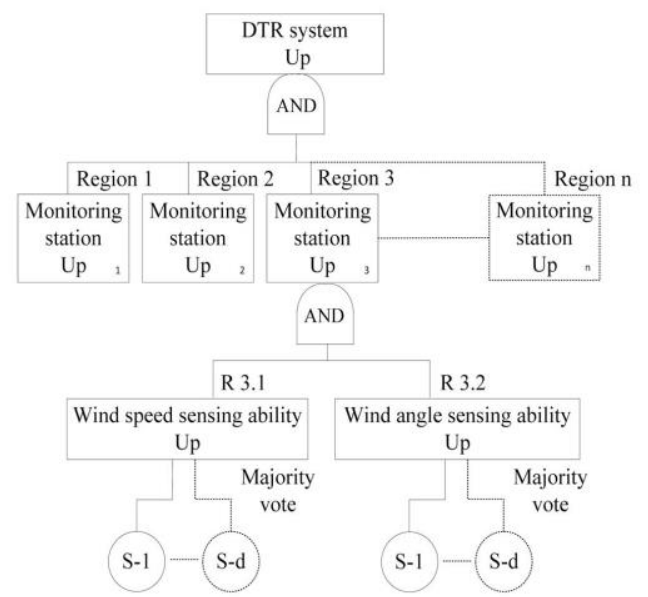

Fig. 2 ETA of DTR system

station. Finally, a DTR system is normal if all the monitoring stations operate normally.

According to the ETA in Fig. 2 and its workflows, the reliability of DTR system is calculated as in (1)

$$
P_{\mathrm{DTR}}^{\mathrm{Up}}=\prod_{i=1}^{n} P_{\text {Region } i}
$$

where

$$
\begin{gathered}
P_{\text {Region } i}=P_{R i .1} \cdot P_{R i .2} \\
P_{R i .1,2}=\sum_{i=(d / 2)+1}^{d}\left(\begin{array}{l}
d \\
i
\end{array}\right)\left(P_{\mathrm{S}}^{\mathrm{Up}}\right)^{i}\left(P_{\mathrm{S}}^{\text {Down }}\right)^{d-i}
\end{gathered}
$$

The symbol $d$ is the total number of wind speeds or wind angle sensors.

\subsection{Sensitivity analyses model}

Statistical sensitivity analysis methods are divided into two groups. The first and the second groups investigate the worth of preserving and improving, respectively, the component reliability so that the system risk can be maintained and lowered, accordingly. Risk achievement (RA), RA worth and Birnbaum importance belong to the first group. The second group constitutes the risk reduction, Fussel-Vesely, and risk reduction worth (RRW) [28-30]. The objective of the second group is relevant to the objective of the proposed framework and among its measures, RRW is commonly utilised [30]. Thus, RRW is chosen as the preferred method for sensitivity analyses.

RRW measures the change in the reliability of the DTR system when the failure rate of a component is set to zero (made perfect) while holding all other inputs at their base value. The importance of a component is proportional to its RRW index. Mathematically, RRW of an arbitrary component $j$ is stated as in (2)

$$
I_{j}^{\mathrm{RRW}}=\frac{P_{\mathrm{DTR}}^{\mathrm{Up}}\left[\lambda_{\text {base }}\right]}{P_{\mathrm{DTR}}^{\mathrm{Up}}\left[\lambda_{\text {base }} \mid \lambda_{j}=0\right]}
$$

Besides RRW, wide range method (WRM) is utilised as well. WRM does not normally have a mathematical expression. Instead, it uses an engineering approach and is performed by varying the reliability of a component one at a time to observe the effect on the reliability of DTR system. As a result, a more critical component will cause a wider swing in the DTR system reliability. In this paper, RRW and WRM are used to have a mixture of statistical and engineering approach of importance measures so that they validate each other.

\section{Assessing network benefits of DTR system}

4.1 Power flow mode

DC-OPF [31] was used to evaluate the impact of implementing a DTR system in the RTN. Although DC-OPF ignores reactive power and voltage constraint, it is faster due to its linear formulation and is widely used in composite reliability analyses $[31,32]$. Transmission network $N-2$ contingency was considered and the generators were set to be fully reliable as their high failure rates will mask the network loss of load. The DC-OPF was implemented using MATPOWER [33]. Mathematically, it is stated as in (3)

$$
\min \left(\mathrm{LC}=\sum_{\forall i \in \mathrm{LB}} \mathrm{LC}_{i}\right)
$$

Subject to

$$
\begin{gathered}
\sum_{\forall j \in \mathrm{GB}} P G_{j}+\sum_{\forall i \in \mathrm{LB}} \mathrm{LC}_{i}=\sum_{\forall i \in \mathrm{LB}} \mathrm{PD}_{i} \\
\mathrm{PG}_{j}^{\min } \leq \mathrm{PG}_{j} \leq \mathrm{PG}_{j}^{\max }, \quad \forall j \in \mathrm{GB} \\
0 \leq \mathrm{LC}_{i} \leq \mathrm{PD}_{i}, \quad \forall i \in \mathrm{LB} \\
\sum_{\forall i \in B_{l}} \frac{\vartheta_{l}-\vartheta_{i}}{X_{l i}}=\mathrm{PG}_{l}-\mathrm{PD}_{l}+\mathrm{LC}_{l}, \quad \forall l \in B
\end{gathered}
$$

On a side note, MC simulation was used to explore the many possible combinations of RTN components and DTR system statuses. The simulation stops and convergence is accepted when the error of RTN indices (i.e. EENS) is $<5 \%$.

\subsection{DTR line rating model}

The IEEE Standard 738 was used to develop the DTR line rating model in this paper. In short, the standard presents the steady-state line rating as a function of weather conditions such as wind speed, wind angle, ambient air temperature, solar radiation angle and conductor physical properties [15]. These weather conditions are measured by monitoring stations located along the transmission line. The formulation of the line rating model is given in (4)

$$
Q_{\mathrm{ci}}\left(T_{\mathrm{c} i}, T_{\mathrm{a} i}, V_{\mathrm{w} i}, \phi_{i}\right)+Q_{\mathrm{ri}}\left(T_{\mathrm{c}}, T_{\mathrm{ai}}\right)=Q_{\mathrm{si}}\left(\theta_{i}\right)+I_{i}^{2} R\left(T_{\mathrm{ci}}\right)
$$

Equation (4) is based on the concept of thermodynamic balance between heat absorbing and releasing rate of the line segment $i$. By rearranging (4), conductor currents are calculated as in (5)

$$
I_{i}=\sqrt{\left(Q_{\mathrm{c} i}+Q_{\mathrm{ri}}-Q_{\mathrm{s} i}\right) / R\left(T_{\mathrm{c} i}\right)}
$$

Finally, the overall DTR line rating is the minimum rating from all line segments, stated as in (6)

$$
I=\min \left(I_{1}, I_{2}, \ldots, I_{n}\right)
$$

\subsection{Weather estimation model}

A MLR model was proposed to estimate the missing weather value of all the non-functional sensors. It is a statistical tool that models the 

Table 1 Reliability data and the number of DTR sensors of a base case
DTR system

\begin{tabular}{lll}
\hline reliability data DTR sensor (occ/year) & $\lambda$ & $\mu$ \\
number of sensors & 3 & 273 \\
\hline
\end{tabular}

relationship between variables (weather data) that are related in a non-deterministic way by using the least-squared estimation (LSE) technique [34]. Generally, it takes on the form as shown in (7)

$$
y=\beta_{0}+\beta_{1} x_{1}+\beta_{2} x_{2}+\cdots+\beta_{n} x_{n}
$$

Relating the equation back to this paper, $y$ are the missing wind speed/angle values of a particular weather region known as the response region and $\left(x_{1}, x_{2}, \ldots, x_{n}\right)$ are the available wind speed angle data in a region known as the regressor regions and are used to predict $y$. All the regressor regions were selected based on a high correlation of weather data with the response region. This selection process is illustrated in the results section. Finally, $\beta$ is the coefficient of regression that is obtained by solving the simplified parallel LSE equations as shown in (7a) (see (7a))

The value $k$ is the size of the sampled weather data.

\subsection{Risk evaluation model}

Risk is defined as the product between the impact of an event and the probability of that event to occur [31]. The impacts that are considered in this paper are loss of load and line annealing. Loss of load is induced by the failure of DTR system and during low DTR ratings under high loading conditions. Line annealing is due to line over-rating. The total risk of the DTR system is given by both events and is stated as in (8)

$$
\text { Risk }_{\text {DTR }}=\text { LOLP }_{\text {DTR }} \times \text { Impact }_{\text {load }}+\mathrm{AP} \times \text { Impact }_{\text {Anl }}
$$

AP is the probability of a line to experience annealing.

The impact of loadloss is the product between the EENS and the cost per megawatt hour (MWh) stated in (8a)

$$
\text { Impact }_{\text {load }}=\text { EENS } \times \text { Cost }_{\text {loadloss }}
$$

The impact of line annealing is the product between the loss of tensile strength (LoTS) due to annealing and the cost of line annealing, stated in $(8 \mathrm{~b})$

$$
\text { Impact }_{\mathrm{Anl}}=\text { LoTS } \times \text { Cost }_{\mathrm{Anl}}
$$

LoTS is expressed as a percentage of its initial strength and is determined using the Harvey model stated as in (9) [35]

$$
\text { LoTS }=100-R S
$$

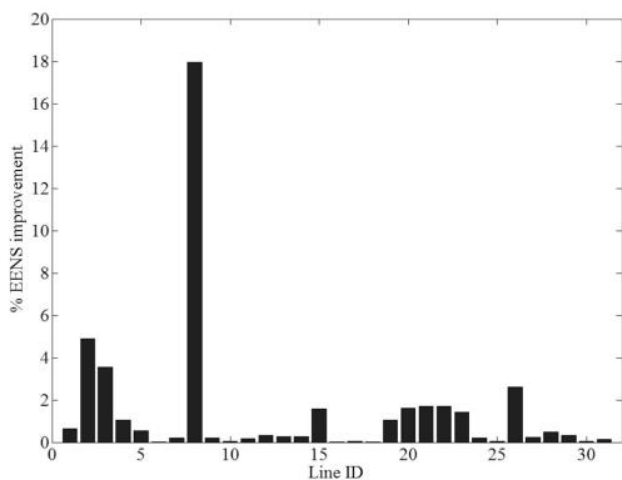

Fig. 3 Percentage improvement in system EENS

where

$$
\mathrm{RS}=\operatorname{RSAl}\left(\frac{\mathrm{STR} \mathrm{Al}}{\operatorname{STR} T}\right)+100 \times\left(\frac{\operatorname{STR~ST}}{\operatorname{STR} T}\right) \times 1.09
$$

$(\operatorname{see}(9 b))$

$\mathrm{STR} T=\mathrm{STR} \mathrm{Al}+\mathrm{STR} \mathrm{ST}=\frac{\pi}{4}\left(n_{\mathrm{Al}} \cdot d_{\mathrm{Al}} \cdot S_{\mathrm{Al}}+n_{\mathrm{ST}} \cdot d_{\mathrm{ST}} \cdot S_{\mathrm{ST}}\right)$

\section{Numerical results and discussion}

\subsection{Description of the test system}

The reliability data and the number of DTR sensors in a base case DTR system design are shown in Table 1 It shows that the initial design has two wind speeds and two wind angle sensors. The risk of the DTR system was assessed in the IEEE 24-bus RTN. The line between bus 7 and bus 8 of the RTN was selected for the DTR system application as it displayed the highest EENS improvement after uprating as shown in Fig. 3. This line is also known as the DTR line. Furthermore, 20 years hourly historical weather data of wind speed, wind angle and air temperature were obtained from the British Atmospheric Data Centre website [36]. The weather data were sampled from 25 weather regions spaced $\sim 5$ miles apart and they are assumed to spread along the DTR line. Hence, the proposed DTR model has 25 monitoring stations. Owing to the high reliability of the RTN, the generation sources and loading levels were increased five-fold in order to highlight the effect of having various designs of the DTR system.

Moreover, an aluminium conductor steel reinforced (ACSR) Lapwing conductor was assumed for the DTR line and it has diameter of $28.1 \mathrm{~mm}$. The line was also assumed to be a straight

$$
\begin{aligned}
& \sum_{i=1}^{k} y_{i} \quad=k \beta_{0}+\beta_{1} \sum_{i=1}^{k} x_{i 1}+\beta_{2} \sum_{i=1}^{k} x_{i 2}+\cdots+\beta_{n} \sum_{i=1}^{k} x_{i n} \\
& \sum_{i=1}^{k} x_{i 1} y_{i}=\beta_{0} \sum_{i=1}^{k} x_{i 1}+\beta_{1} \sum_{i=1}^{k} x_{i 1}^{2}+\beta_{2} \sum_{i=1}^{k} x_{i 1} x_{i 2}+\cdots+\beta_{n} \sum_{i=1}^{k} x_{i 1} x_{i n}
\end{aligned}
$$

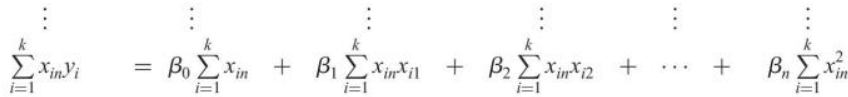

$$
\mathrm{RS} \mathrm{Al}=\left\{\begin{array}{cc}
\left(-0.24 T_{\mathrm{c} i}+134\right) t^{-\left(0.001 T_{\mathrm{ci}}-0.095\right)\left(0.1 / d_{\mathrm{Al}}\right),} & \text { if }\left(-0.24 T_{\mathrm{c} i}+134\right)<100 \\
100, & \text { otherwise }
\end{array}\right\}
$$


Table 2 RRW analysis on monitoring station and DTR system reliability

\begin{tabular}{lcc}
\hline Cases & Monitoring station & DTR system \\
\hline base case & 0.9572 & 0.3352 \\
perfect wind speed sensors & $0.9784(+2.2 \%)$ & $0.5790(+72.7 \%)$ \\
perfect wind angle sensors & $0.9784(+2.2 \%)$ & $0.5790(+72.7 \%)$ \\
\hline
\end{tabular}

line running in an East-West direction. In short, the azimuth of the line is $90^{\circ}$. In other lines that are not equipped with the DTR system, the pre-fault and post-fault STRs given by the RTN were adopted. The STR of the DTR line was calculated as 236 MVA and the conservative weather assumptions used for the STR calculation are $0.6 \mathrm{~m} / \mathrm{s}$ wind speed, $0^{\circ}$ wind angle, $40^{\circ} \mathrm{C}$ ambient temperature and solar radiation angle at $12 \mathrm{pm}$.

\subsection{Reliability assessment of DTR system}

Using the proposed reliability model, the reliability of the monitoring station in each region and the DTR system were calculated as 0.9572 and 0.3352 , respectively. To explore other designs, RRW and WRM were performed to determine the critical components of the DTR system. The results for RRW and WRM are shown in Table 2 and Fig. 4, respectively. In Table 2, the results show that wind speed and wind angle sensors are equally critical in the reliability of the DTR system. In Fig. 4, the reliability of the monitoring station and the DTR system responds in the same manner when the reliability of the wind speed and wind angle sensors was varied individually. The variation was made by multiplying the failure rate of the sensors according to the scale that is shown in this figure. It was observed that the results from Table 2 and Fig. 4 agree with each other and all design works should be performed on both types of sensors. Consequently, the three following designs were considered for wind speed and wind angle sensors:

Design (A): Add redundancy.

Design (B): Reduce failure rates on top of option A.

Design $(C)$ : Reduce failure rates and shorten repair times on top of option A.

The effects of exploring Design A are shown in Fig. 5. It shows that the reliability of the monitoring station increased by $4.4 \%$ from 0.9572 to 0.9993 when the number of sensors was increased from 2 to 3 . However, the DTR system shows an improvement in its reliability from 0.3352 to 0.9826 . Redundancy enhancements achieved through the use of more than three sensors were insignificant due to saturation of reliabilities as they approached toward the perfect state. Beyond this point, an even number of sensors provided lower reliability than odd number of sensors. The reason is because the majority vote system requires an even

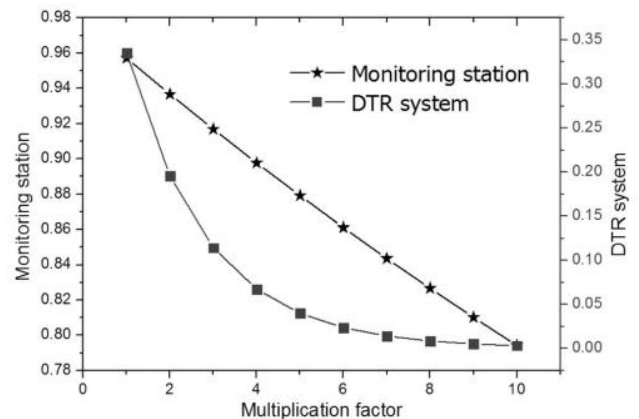

Fig. 4 WRM analysis on monitoring stations and DTR system reliability

IET Gener. Transm. Distrib., pp. 1-8

C) The Institution of Engineering and Technology 2015

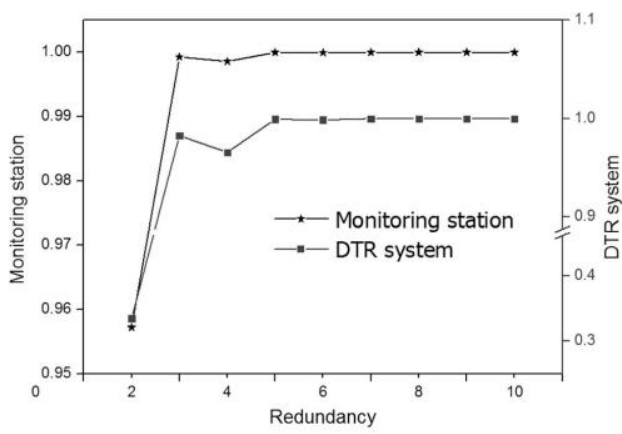

Fig. 5 Reliability of DTR system design option A

number configuration to have more functional sensors than odd number configuration in order to have normal sensing abilities. For example, a three- and four-sensor design requires 67 and $75 \%$ of functional sensors, respectively, in order to yield a normal sensing ability. This shows that the number of redundancy is not always directly proportional to the DTR system reliability. From the perspective of a DTR system planner, cost can be saved by not investing in the wrong number of DTR sensors. Designs B and C were explored and their responses are shown in Fig. 6. During the analyses, failure and repair rates of wind speed and wind angle sensors were scaled with factors of 1-0.02. As expected, Design C is superior to Design B.

\subsection{Robustness of weather estimation model}

In this section, the robustness of the proposed MLR model is demonstrated. First, the set-up of the model is described. The correlations of wind speed and wind angle among the 25 regions were determined and it was found that wind speed generally has higher correlation among the regions than wind angle. Hence, the correlation levels of 95 and $90 \%$ were selected to qualify a candidate region as a wind speed and wind angle regressor region, respectively. The qualified pairs of regressor and response regions are shown in Table 3. Although a higher correlation level was needed to qualify as wind speed regressor region, the member in this group is significantly more than that of wind angle. In each region, weather data from at least one regressor region is needed to estimate the missing wind speed or wind angle. If all sensors in all the regressor regions are unavailable, the weather data cannot be estimated and STR would be assumed.

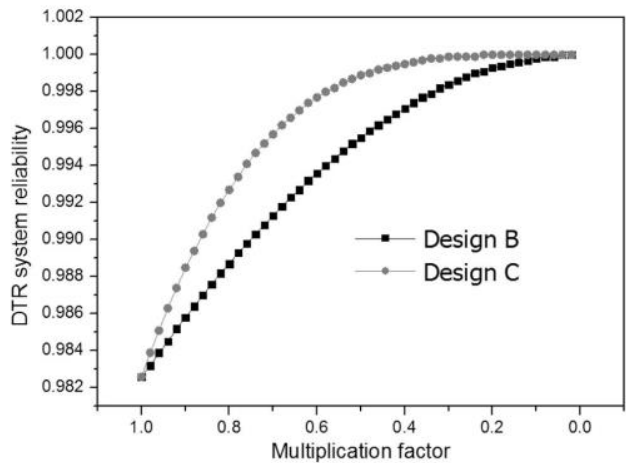

Fig. 6 Reliability of DTR system design options B and C 
Table 3 Wind speed, wind angle regressor and response region pairs

\begin{tabular}{lcc}
\hline Regions & Wind speed regressor regions & $\begin{array}{c}\text { Wind angle regressor } \\
\text { regions }\end{array}$ \\
\hline 1 & $2,3,4,5,6$ & 2,3 \\
2 & $1,3,4,5,6,7$ & $1,3,4$ \\
3 & $1,2,4,5,6,7,8$ & $1,2,4,5$ \\
4 & $1,2,3,5,6,7,8,9$ & $2,3,5,6$ \\
5 & $1,2,3,4,6,7,8,9,10$ & $3,4,6,7,8$ \\
6 & $1,2,3,4,5,7,8,9,10,11$ & $4,5,7,8,9$ \\
7 & $2,3,4,5,6,8,9,10,11,12$ & $5,6,8,9$ \\
8 & $3,4,5,6,7,9,10,11,12,15$ & $5,6,7,9,10$ \\
9 & $4,5,6,7,8,10,11,12,15,16$ & $6,7,8,10,11$ \\
10 & $5,6,7,8,9,11,12,15,16,17$ & $8,9,11,12$ \\
11 & $6,7,8,9,10,12,15,16,17,18$ & $9,10,12$ \\
12 & $7,8,9,10,11,13,14,15,16,17,18$ & $10,11,15,16$ \\
13 & $12,14,15,16,17,18,19,20,21,22$ & $14,17,18,19$ \\
14 & $12,13,15,16,17,11,19,20,21,22,23$ & $13,17,18,19$ \\
15 & $8,9,10,11,12,13,14,16,17,18$ & $12,16,17$ \\
16 & $9,10,11,12,13,14,15,17,18,19,20$ & $12,15,17,18$ \\
17 & $10,11,12,13,14,15,16,18,19,20,21$ & $13,14,15,16,18$ \\
18 & $11,12,13,14,15,16,17,19,20,21,22$ & $13,14,16,17,19$ \\
19 & $13,14,16,17,18,20,21,22,23,24$ & $13,14,18,20,21$ \\
20 & $13,14,16,17,18,19,21,22,23,24,25$ & $19,21,22$ \\
21 & $13,14,17,18,19,20,22,23,24,25$ & $19,20,22,23$ \\
22 & $13,14,18,19,20,21,23,24,25$ & $20,21,23,24$ \\
23 & $14,19,20,21,22,24,25$ & $21,22,24,25$ \\
24 & $19,20,21,22,23,25$ & $22,23,25$ \\
25 & $20,21,22,23,24$ & 23,24 \\
\hline
\end{tabular}

There are two types of regressions in the proposed MLR weather estimation model - complete and incomplete. The former is defined as the case in which weather data in all the regressor regions are available. Incomplete regression is the case where weather data from at least one but not all the regression regions are missing (sensor in non-functional state). Weather estimations of the following three scenarios were investigated for their accuracy in all regions:

Scenario (1): Wind speed estimation.

Scenario (2): Wind angle estimation.

Scenario (3): Wind speed and wind angle estimations.

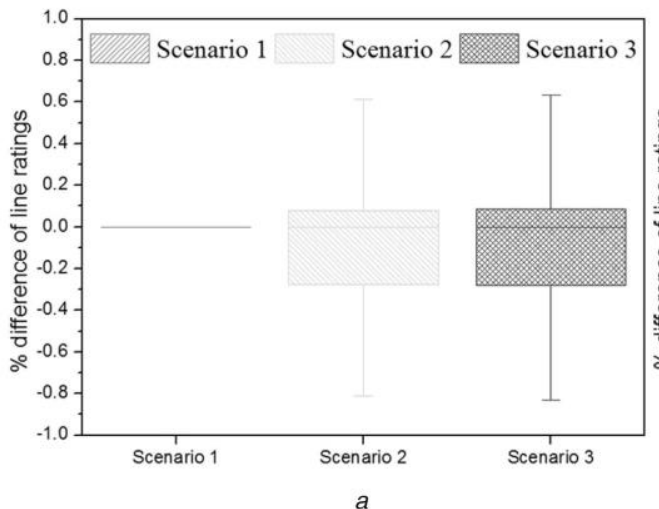

To ensure that the results in each scenario are fully representative of the MLR model performance, all the combinations of cases in each scenario were exhausted. For complete regressions, there are 25 possible combinations of cases in each scenario. Incomplete regressions have $24,206,398$ and 443,492 numbers of cases in each scenario, respectively. The estimated weather data were used to calculate the estimated line ratings and the differences from actual line rating values (calculated from actual weather data) were determined. The findings of all cases of the same scenario and regression type were consolidated. The results of complete and incomplete regressions are shown as the boxplot in Figs. $7 a$ and $b$, respectively.

Fig. $7 a$ shows that complete regression of Scenario 1 yields negligible percentage differences between the estimated and actual line ratings. Complete regression of Scenario 2 produces a slightly higher percentage difference due to the lower correlation of wind angle among the regions. The percentage differences are between -0.8 and $0.6 \%$. A positive value indicates that the estimated line ratings are higher than the real ratings and vice versa. Owing to the negligible effects of Scenario 1, the results of Scenario 2 and 3 are similar. As transmission assets are expensive and sensitive toward thermal overload, the line ratings estimated in Scenarios 2 and 3 should be reduced by at least $0.6 \%$ to avoid over-rating. In this paper, a slightly conservative value of $1 \%$ was adopted. Line ratings estimated from Scenario 1 do not require reduction due to negligible differences from real line ratings.

Similarly, Fig. $7 b$ shows that the percentage differences in the line ratings are higher in Scenario 2 than in Scenario 1. Hence, estimation errors of wind speed have only little influence in Scenario 3. As expected, incomplete regression yields a higher percentage difference than complete regression across all the scenarios. For the same security reasons as mentioned previously, estimated line ratings obtained through incomplete regression in Scenarios 1,2 and 3 should be reduced by at least $0.64,4.5$ and $5.32 \%$, respectively. In this paper, we adopted the slightly more conservative values of 1,5 and $6 \%$, respectively.

Both of the results demonstrated that accurate MLR weather estimation models were constructed by considering only the strong correlations of wind speed and wind angle among the weather

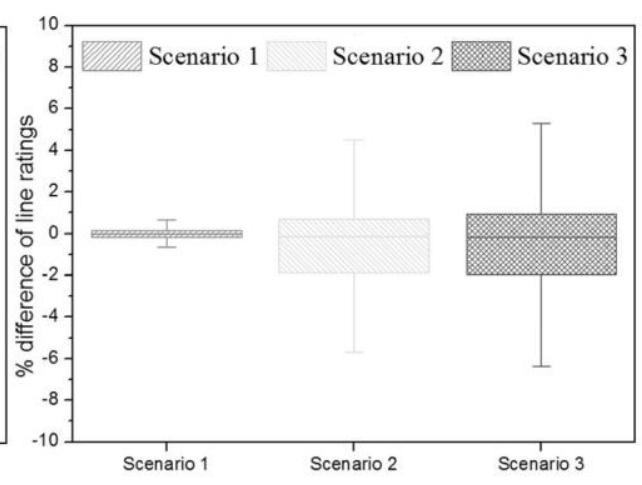

Fig. 7 Robustness of weather estimation model

$a$ Complete regression

$b$ Incomplete regression: Boxplot of percentage differences between estimated and actual line ratings for scenarios 1, 2 and 3

Table 4 Average VoLL for various conditions

\begin{tabular}{|c|c|c|c|c|c|c|}
\hline \multirow[t]{2}{*}{ Average interruption cost of $1 \mathrm{~h}, \mathrm{E} / \mathrm{MWh}$} & \multicolumn{3}{|c|}{ Winter } & \multicolumn{3}{|c|}{ Summer } \\
\hline & $\begin{array}{l}\text { Peak (3-9 pm) } \\
6495.69\end{array}$ & $\begin{array}{l}\text { Off peak } \\
6525.70\end{array}$ & $\begin{array}{l}\text { Weekend } \\
5634.43\end{array}$ & $\begin{array}{l}\text { Peak (3-9 pm) } \\
6268.91\end{array}$ & $\begin{array}{l}\text { Off peak } \\
5727.02\end{array}$ & $\begin{array}{l}\text { Weekend } \\
5178.49\end{array}$ \\
\hline
\end{tabular}

IET Gener. Transm. Distrib., pp. 1-8 
Table 5 Risk of various DTR system design options

\begin{tabular}{|c|c|c|c|c|c|c|}
\hline \multirow[t]{2}{*}{ Scenarios } & \multicolumn{3}{|c|}{ With weather estimation model } & \multicolumn{3}{|c|}{ Without weather estimation model } \\
\hline & $\begin{array}{l}\text { Risk of loadloss, } \\
\text { GE/year }\end{array}$ & $\begin{array}{l}\text { Risk of annealing, } \\
\text { kf/year }\end{array}$ & $\begin{array}{l}\text { Total risk, } \\
\text { Gf/year }\end{array}$ & $\begin{array}{l}\text { Risk of loadloss, } \\
\text { Gf/year }\end{array}$ & $\begin{array}{l}\text { Risk of annealing, } \\
\text { kf/year }\end{array}$ & $\begin{array}{l}\text { Total risk, } \\
\text { GE/year }\end{array}$ \\
\hline base case & 1.9505 & 0 & 1.9505 & 4.4162 & 645.49 & 4.4162 \\
\hline $\operatorname{design} \mathrm{A}$ & 1.9420 & 0 & 1.9420 & 4.4113 & 644.14 & 4.4113 \\
\hline design B & 1.9404 & 0 & 1.9404 & 4.4012 & 642.50 & 4.4012 \\
\hline design C & 1.8882 & 0 & 1.8882 & 4.2560 & 642.46 & 4.2560 \\
\hline less DTR & 3.6544 & 1.0261 & 3.6544 & 5.4589 & 645.50 & 5.4589 \\
\hline
\end{tabular}

regions. Line ratings that were estimated via complete regression were close to actual values in all of the scenarios. The MLR models are also robust against the loss of its regressor regions. models are also robust against the loss of its regressor regions.
This was reflected by the containment of highest estimated line ratings error to within $6 \%$ in the incomplete regression scenarios.

\subsection{Risk assessment of DTR system}

All the DTR system designs suggested in Section 6.2 were subjected to risk assessment. For comparison, their risk values were determined with and without the use of the proposed MLR model. Moreover, the risk of a base case DTR system and a DTR system with its sensor reliability reduced by ten-fold (indicated as less DTR) were examined as well.

During the risk assessment, the average values of loadloss (VoLLs) as shown in Table 4 were used. These values were taken from the report of the Royal Academy of Engineering for the UK Prime Minister's Council of Science and Technology [37]. As fo the cost of annealing, conductor replacement is considered necessary for every $100 \%$ of conductor annealing. Using the data sheet published by the Southwire company [38], an ACSR Lapwing conductor, the conductor of choice for our DTR line, is weighted as $5872.7 \mathrm{lbs} / \mathrm{km}$ and is priced at $1.81 / \mathrm{lbs}$. Given a 75 $\mathrm{km}$ DTR line as stated in the RTN, the total weight of the conductor was calculated as 440 klbs (rounded value) and the price of replacing the conductor was valued at $797,000 / 100 \%$ annealing.

Finally, by using (8) and (9), the risks are calculated and are shown in Table 5. In all the scenarios, the risks of loadloss determined without weather estimation are higher than when weather is estimated. In the former case, when the DTR sensor are not functional, the line ratings revert to STR and waste huge current carrying capacity that lead to more loadloss than the latter case. Moreover, actual line ratings are sometimes lower than the STR and lead to more cases of conductor overrating and higher risk of annealing. Overall, the results clearly indicate that the proposed MLR model avoids overestimating the DTR system risk.

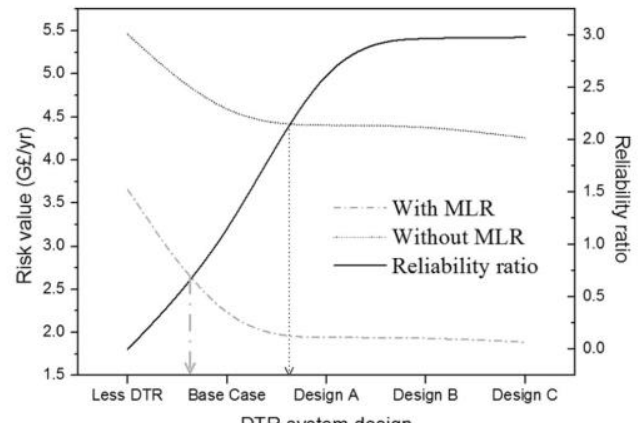

DTR system design

Fig. 8 Reliability and risk curve of DTR system with and without MLR weather estimation function
Owing to the accuracy of the MLR weather estimation model, its se avoided line annealing in all the scenarios except for the "less DTR' scenario. Line annealing persists in this scenario as its DTR' scenario. Line annealing persists in this scenario as its
sensors have very low reliability and are prone to failure. In some cases, weather cannot be estimated due to the loss of all regressor sensors subsequently forcing the STR to be accepted. Hence, the risk of annealing is not due to the inaccuracy of the MLR model. Rather, it is the result of unreliable DTR sensors.

Finally, optimisation between the reliability and the risk of all the DTR system designs was performed and the results are as shown in Fig. 8. Note that reliability is expressed in the form of a ratio by comparing other designs with the 'base case' DTR system, which also represents the scale of DTR system costs. Moreover, two types of risk values are displayed in this figure - one for the case with MLR weather estimation and the other without. Moreover, shown in this figure are the intersections between the reliability line and risk lines that indicate the optimum DTR system reliability. The results show that the optimum DTR system reliability is lower when it is equipped with the MLR weather estimation function. These results concur with Table 5 that the proposed weather estimation model avoids overestimating the risk of the DTR system. In other words, given the same load level, the use of an MLR weather estimation function can accommodate a less reliable DTR system while lowering its risk impact. Note that the optimum DTR system designs have reliability values that are not exactly the same as those that are presented in Fig. 8 due to the smoothing of the lines during plotting. Hence, the next available choice was suggested as the optimum design. In the case where MLR weather estimation was used, the 'base case' design was found to be the optimum whereas 'Design A' is the optimum for the case without MLR weather estimation.

\section{Conclusions}

One of the main concerns of DTR system deployment is to ensure it operates properly at all times with minimal risk to the power network. To address this, this paper presented a framework that assesses the integrity (reliability and risk) of a DTR system. As a bottom line, three contributions are concluded from the presented bottom

First, a framework that assesses the reliability and risk of DTR system was proposed. Part 1 of the framework explores various DTR system designs and evaluates their reliabilities. In Part 2, the risk values of all the designs are assessed. Then, the optimum DTR system design is selected based on the optimisation between the risk and reliability values.

Second, this paper proposed an MLR weather estimation model, as reverting to STR during DTR sensor failures was found to overestimate the risks of the DTR system. The weather estimation model was proven to be accurate and can estimate line ratings close to the actual values. This allows more loads to be served and reduces line overrating occurrence. It is also robust against the loss of multiple weather data. As a result of this model, a less reliable DTR system can be used while lowering its risk to the power network.

Finally, the analyses of DTR system reliability pointed out that having more DTR sensor redundancies is not necessarily better for the reliability of the DTR system. In particular, when the majority

IET Gener. Transm. Distrib., pp. 1-8 
vote system is in use, an odd number of DTR sensor configurations offer more advantages than an even number configuration, that is, three sensors and four sensors. This is important to avoid over and under-deployment of the sensors.

\section{Acknowledgments}

The authors thank the Universiti Sains Malaysia (USM) and the Ministry of Higher Education Malaysia (MOHE) for the funding support.

\section{References}

I Cigre Brochure: 'Guide for the selection of weather parameters for bare overhead conductor ratings'. 2006

2 Holbert, K.E., Heydt, G.T.. Prospects for dynamic transmission circuit ratings'. The 2001 IEEE Int. Symp. Circuits and Systems, 2001, (ISCAS 2001), 2001, vol. 2, pp. 205

3 Davis, M.W.: 'A new thermal rating approach: the real time thermal rating system for strategic overhead conductor transmission lines - part I: general description an justification of the real time the

Dars, M.W.: 'A new thermal rating approach: the real time thermal rating systen Weread conductor transmission lines - part II: steady state therma rating program', IEEE Trans. Power Appar. Syst., 1977, 96, pp. 810-825

5 Davis, M.W.: 'A new thermal rating approach: the real time thermal rating systen for strategic overhead conductor transmission lines part III steady state therma rating program continued-solar radiation considerations', IEEE Trans. Power Appar. Syst., 1978, PAS-97, pp. $444-45$

Davis, M.W.: 'A new thermal rating approach: the real time thermal rating syster for strategic overhead conductor transmission lines part IV daily comparisons of real-time and conventional thermal rating and establishment of typical annua

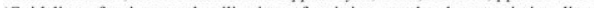
CIGRE WOKing Group B2.13, 2008 - 2 .

Pitchard, G.A.: 'Field application of a dynamic thermal circuit rating method', IEEE Trans. Power Deliv., 1997, 12, pp. 823-831

9 Douglass, D.A., Edris, A.: 'Field studies of dynamic thermal rating methods for pp. 842-851

0 Douglass, D.A., Edris, A.: 'Real-time monitoring and dynamic thermal rating of power transmission circuits, ILEE Trans. Power DelN., 1996, 11, pp. 1407-1418 1 Seppa, O., Clements, M., Damsgaard-Mikkelsen, S., et al.. 'Application of rea time thermal ratings for optimizing transmission line investment and operatin

2 Seppa, T.O.: 'Increasing transmission capacity by real time monitoring'. IEEF

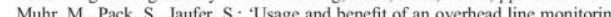
system'. It Conf on High Voltoge Engineering and Application (ICOHVE). 2008 , pp. 557-561
14 Yi, Y., Lambert, F., Divan, D.: 'A survey on technologies for implementing sensor networks for power delivery systems'. IEEE Power Engineering Society General Meeting for power delich

15 'IEEE Standard for Calculating the Current-Temperature of Bare Overhead Conductors', IEEE Std. 738-2006 (Revision of IEEE Std. 738-1993), 2007, pp. cl-c59

16 Tumelo-Chakonta, C., Kopsidas, K. ' Assessing the value of employing dynamic thermal rating on system-wide performance'. Second IEEE PES Int. Conf. and Exhibition on Innovative Smart Grid Technologies (ISGT Europe), 2011, pp. 1-8 Matus, M., Saez, D., Favley, M., et al.: 'Identification of critical spans for monitoring systems in dynamic thermal rating', IEEE Trans. Power Deliv. 2012, 27, pp. 1002-1009

18 Chu, R.F.: 'On selecting transmission lines for dynamic thermal line rating system

Shaker, H., Fotuhi-Firuzabad, M., Aminifar, F.: 'Fuzzy dynamic thermal rating of transmission lines', IEEE Trans. Power Deliv., 2012, 27, pp. 1885-1892

年, thermal rating', IEEE Trans. Power Deliv, 2013, 28, pp. 1600-1609 1 Siler, M., Heckenbergerova, J., Musilek, P., et al.: 'Sensitivity analysis of on Electrical and Computer Engineering (CCECE, 2013), 2013, pp. 1-4

22 Billinton, R., Allan, R.N.: 'Reliability evaluation of engineering systems: concepts and techniques' (Longman Scientific \& Technical, Harlow, 1987)

23 Lee, K.Y., Chan-Eom, P.: 'Sensor value validation based on implicit sensor redundancy for reliable operation of power plants', IEEE Trans. Energy Chow, E. Willsky, A S. 'Anely tic

nalytical redundancy and the design of robust failure detection systems', IEEE Trans. Autom. Control, 1984, 29, pp. 603-614

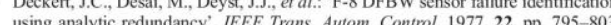

Deyst, JJ, Kanazawa, R.M., Pasquenza, J.P. 'Sensor validation: a method to enhance the quality of the man/machine interface in nuclear power stations', IEEE Trans. Nucl. Sci., 1981, 28, pp. 885-890

27 lyengar, S.S., Prasad, L.: 'A general computational framework for distributed sensing and fault-tolerant sensor integration', IEEE Trans. Syst. Man Cybern., 1995, 25, pp. 643-650

Modarres, M., Kaminskiy, M., Krivtsov, V. 'Reliability engineering and risk analysis: a practical guide' (Marcel Dekker Inc., New York, Basel, 1999)

GeE Guide for Gencral Principles of Reliability Analysis of Nuclear Powe van de Borst $\mathrm{M}$, Scho Reliab. Eng. Syst. Saf., 2001, 72, pp. 241-245

Wenyuan, L.: 'Risk assessment of power system' (IEEE-Wiley, 2005)

32 Billinton, R., Allan, R.N.: 'Reliability evaluation of power systems' (Plenum, New York, 1984)

Zimmerman, R.D., Murillo-Sánchez, C.E., Thomas, R.J.: 'MATPOWER: steady-state operations, planning, and analysis tools for power systems research and education, IEEE Trans. Power Sys., 2011, 26, pp. 12-19

34 Montgomery, D.C., Runger, G.C.:. 'Applied statistics and probability for engineers'

35 Harvey, J.R.: 'Effect of elevated temperature operation on the strength of aluminum conductors, IEEE Trans. Power Appar. Syst., 1972, PAS-91, pp. 1769-1772

37 'Counc. (BADC). [One UK'. Rotal Academy of Engineering November 2014

38 Southwire: Available at http://www.southwire.com/Ordering.htm 